\title{
Cefaléias Secundárias na Infância
}

\author{
Deusvenir de Souza Carvalho*
}

\section{RESUMO}

As cefaléias secundárias na infância são abordadas segundo os principais itens da classificação de 1988 da International Headache Society. O conceito de cefaléia secundária ou sintomática, bem como a visão de peculiaridades dos aspectos clínicos para o diagnóstico na infância são apresentados. São feitos breves comentários da orientação aos casos.

\section{UNITERMOS}

Cefaléias na infância, cefaléias secundárias na infância, cefaléias sintomáticas na infância.

\section{NTRODUÇÃO}

Cefaléia é um sintoma. Não perdendo de vista esse horizonte, pode-se encontrar dois grandes grupos nos quais ela aparece: o grupo das cefaléias primárias e o grupo das cefaléias secundárias ou sintomáticas. As cefaléias primárias são aquelas em que cefaléia é o sintoma principal na doença ou na síndrome e no caso das secundárias ou sintomáticas, a doença ou síndrome é outra quando uma das manifestações é o sintoma cefaléia.

A Classificação e Critérios Diagnósticos das Cefaléias, Nevralgias Cranianas e Dor Facial, proposta em 1988 pelo Comitê de Classificação das Cefaléias da Sociedade Internacional de Cefaléia (IHS) ${ }^{1}$, enumera as cefaléias primárias nos itens de 1 a 4 e as cefaléias secundárias ou sintomáticas, aqui abordadas, nos de 5 a 11, as neuralgias no item 12, restando o item 13 para as cefaléias não classificáveis (Tabela 1). As subdivisões de cada um dos

\section{TABELA 1}

\section{Principais itens da Classificação da IHS ${ }^{1}$}

\section{Cefaléias primárias}

1 - Enxaqueca (ou migrânea)

2 - Cefaléia tipo tensão

3 - Cefaléias em salvas e hemicrânia paroxística crônica

4 - Cefaléias diversas não associadas a lesões estruturais

Cefaléias secundárias ou sintomáticas

5 - Cefaléia associada a trauma de crânio

6 - Cefaléia associada a doenças vasculares

7 - Cefaléia associada a outros distúrbios intracranianos nãovasculares

8 - Cefaléia associada a substâncias ou à sua retirada

9 - Cefaléia associada à infecção não cefálica

10 - Cefaléia associada a distúrbio metabólico

11 - Cefaléia ou dor facial associada a distúrbio do crânio, pescoço, olhos, orelhas, seios paranasais, dentes ou a outras estruturas faciais ou cranianas

12 - Neuralgias cranianas, dor de tronco nervoso e dor de deaferentação

13 - Cefaléia não classificável 
itens são apresentadas no texto, na medida da necessidade. Serão consideradas as principais cefaléias secundárias ou sintomáticas da infância e da adolescência.

\section{CEFALÉIAS SECUNDÁRIAS OU SINTOMÁTICAS}

As cefaléias secundárias ou sintomáticas levantam uma questão intrigante sobre o processo que, por vezes, torna-a semelhante às cefaléias primárias. Por outro lado, o estudo dessas cefaléias secundárias pode ajudar a entender a fisiopatologia e a etiopatogenia das primárias $^{2}$. Não se pretende aprofundar nos quadros específicos, sendo suficiente enfocar os diagnósticos a partir do sintoma cefaléia.

Mesmo quando a queixa principal é a dor de cabeça e o exame geral é normal, ainda é espantoso o número de crianças e de adolescentes que procuram o neurologista ou o especialista em cefaléia, somente depois de consulta ao otorrinolaringologista e/ou ao oftalmologista ${ }^{3,4,5,6,7}$. Um levantamento sobre o ensino médico na Europa mostrou que, durante a graduação, o aluno de medicina recebe informação sobre dor de cabeça durante uma hora apenas ${ }^{8}$. No entanto, essa queixa certamente é feita, pelos pacientes, a maioria, se não a todos os especialistas.

\section{CEFALÉIA ASSOCIADA A TRAUMA DE CRÂNIO}

A classificação e os critérios para a cefaléia associada a trauma de crânio são indicados na tabela 2, deixando bem claro os dados que indicam esses diagnósticos.

Apesar de a cefaléia pós-traumática guardar relação com o trauma craniano, o mecanismo responsável por sua gênese ainda é desconhecido ${ }^{1,9}$.

Após um trauma craniano, que pode ou não ser significativo e acompanhar-se ou não de outros sintomas e sinais comprobatórios ao exame físico ou subsidiário (vide Tabela 2), o paciente pode apresentar cefaléia. Para que esse sintoma seja relacionado ao trauma, ele deve se iniciar até 14 dias a partir do mesmo, podendo durar até 8 semanas (forma aguda), ou persistir por mais de 8 semanas (forma crônica).

\section{TABELA 2}

Classificação e critérios diagnósticos da cefaléia associada a trauma de crânio

Cefaléia pós-traumática aguda com trauma de crânio significativo e/ou sinais comprobatórios

A. A importância do trauma de crânio é documentada por, pelo menos, um dos seguintes itens:

1 - Perda de consciência

2 - Amnésia pós-traumática durando mais de 10 minutos 3 - Pelo menos dois dos seguintes mostrando anormalidades: exames clínico e neurológico, raio $\mathrm{X}$ do crânio, neuroimagem, potenciais evocados, líquido cefalorraquidiano, provas de função vestibular, testes neuropsicológicos

B. A cefaléia ocorre em menos de 14 dias após recuperação da consciência (ou após o trauma, se não houver perda de consciência)

C. A cefaléia desaparece em 8 semanas ou menos, após recuperação da consciência (ou após o trauma, se não houver perda de consciência)

Cefaléia pós-traumática aguda sem trauma de crânio significativo e sem sinais comprobatórios

A. Trauma de crânio que não satisfaz o item A para cefaléia com sinais comprobatórios

B. A cefaléia ocorre em menos de 14 dias após o trauma

C. A cefaléia desaparece em 8 semanas ou menos após o trauma
Cefaléia pós-traumática crônica com trauma de crânio significativo e/ou sinais comprobatórios

A. A importância do trauma de crânio é documentada por, pelo menos, um dos seguintes itens:

1 - Perda de consciência

2 - Amnésia pós-traumática durando mais de 10 minutos

3 - Pelo menos dois dos seguintes mostrando anormalidades: exames clínico e neurológico, raio $\mathrm{X}$ do crânio, neuroimagem, potenciais evocados, líquido cefalorraquidiano, provas de função vestibular, testes neuropsicológicos

B. A cefaléia ocorre em menos de 14 dias após recuperação da consciência (ou após o trauma, se não houver perda de consciência)

C. A cefaléia persiste além de 8 semanas, após recuperação da consciência (ou após o trauma, se não houver perda de consciência)

Cefaléia pós-traumática crônica sem trauma de crânio significativo e sem sinais comprobatórios.

A. Trauma de crânio que não satisfaz o item A para cefaléia com sinais comprobatórios

B. A cefaléia ocorre em menos de 14 dias após o trauma

C. A cefaléia persiste além de 8 semanas, após o trauma 
A cefaléia apresenta-se em torno de $15 \%$ nos pacientes que sofreram trauma craniano. Do total daqueles que sofreram trauma significativo e/ou com sinais comprobatórios (item 3 da Tabela 2), o quadro de cefaléia aparece em torno de $40 \%$ a $60 \%$ dos pacientes ${ }^{10,11}$.

Além da cefaléia, podem-se associar, isolada ou conjuntamente, outros sinais e sintomas, tais como tontura, irritabilidade, ansiedade, vômitos. A forma crônica, mais comum em adultos que em crianças, está inserida no contexto de uma síndrome pós-concussional que apresenta, além da cefaléia, outros sintomas, como distúrbios de memória, distúrbios comportamentais, distúrbios de humor, redução da atenção e do rendimento escolar. Nesses pacientes, os fatores psíquicos devem ser analisados e a abordagem multidisciplinar (psicodiagnóstico) é necessária.

Nos pacientes com cefaléia associada a trauma de crânio, a anamnese e o exame físico geral e neurológico, ainda que muito cuidadosos, podem não ser suficientes para excluir lesão orgânica (ex.: hematoma subdural) que, na maioria dos casos, ocorre nas primeiras 24 horas. Exames subsidiários complementares são necessários nesses casos.

O tratamento sintomático do quadro agudo é feito com analgésicos e antiinflamatórios. $\mathrm{O}$ tratamento profilático é complexo, devido aos fatores psicoemocionais envolvidos. Após o traumatismo craniano, pode surgir uma cefaléia com características de enxaqueca ou de cefaléia tipo tensão ${ }^{12}$.

\section{CEFALÉIA ASSOCIADA A DOENÇAS VASCULARES}

Os critérios para esse diagnóstico são:

- sintomas ou sinais de distúrbio vascular;

- investigações apropriadas que indicam distúrbio vascular;

- cefaléia como um sintoma novo ou com uma nova apresentação estando em clara relação temporal com a instalação do distúrbio vascular.

Estão enquadrados neste grupo: a cefaléia da doença vascular isquêmica aguda, o ataque isquêmico transitório, o episódio isquêmico tromboembólico, o hematoma intracerebral parenquimatoso, o hematoma subdural, o hematoma epidural, a hemorragia subaracnóidea, a malformação arteriovenosa, o aneurisma, as arterites, as tromboses venosas intracranianas, a dissecção da artéria carótida ou da vertebral, a carotidinia, a pós-endarterectomia, a hipertensão arterial aguda e a crônica, o feocromocitoma, a pré-eclâmpsia e a eclâmpsia.

\section{CEFALÉIA ASSOCIADA A OUTROS DISTÚRBIOS INTRACRANIANOS NÃO-VASCULARES}

\author{
Critérios diagnósticos:
}

- Sinais e sintomas de distúrbio intracraniano.

- Confirmação deste por investigação apropriada.

- Cefaléia como um sintomas novo ou como uma nova apresentação ocorre em clara relação com o distúrbio intracraniano.

Os quadros são: hipertensão intracraniana idiopática, hidrocefalia, hipotensão intracraniana, pós-punção lombar, fístula liquórica, infecção intracraniana, meningite, encefalite, abscesso cerebral, empiema subdural, sarcoidose e outras doenças inflamatórias não infecciosas, cefaléia associada à injeção intratecal e às neoplasias.

\section{Tumor cerebral}

A cefaléia secundária a tumores cerebrais pode aparecer na dependência da localização e da velocidade de crescimento da massa intracraniana ${ }^{13}$. A sua suspeita pode ser feita sempre que a história apontar para uma piora progressiva na freqüência, na intensidade ou na duração dessa cefaléia. Em alguns casos de tumores de crescimento lento, a cefaléia pode ser o único sintoma durante meses, sem qualquer sinal de déficit neurológico associado.

O caráter da cefaléia é geralmente não pulsátil, com duração de minutos a horas e localização também variável; o período preferencial é o matutino e inclui como fatores de piora, a atividade física rotineira ou o esforço e manobras que levem a aumento da pressão intracraniana (Valsalva). Pode também, em alguma fase, apresentar como sinais e sintomas acompanhantes aqueles da síndrome de hipertensão intracraniana, ou seja, além da cefaléia, os vômitos, sendo estes às vezes em jato e não precedidos de náuseas, e papiledema. $\mathrm{O}$ exame neurológico pode também apresentar, dependendo da localização do tumor, alterações do estado mental, distúrbios visuais e da fala, ataxia, parestesias e déficits motores. Eventualmente ocorrem crises epilépticas. O tratamento é feito pela abordagem do tumor, o qual pode ser cirúrgico, dependendo das características anatomoclínicas e, em alguns casos, pode haver necessidade de complementação pela quimioterapia e/ou radioterapia.

Embora haja uma grande preocupação, por parte dos responsáveis pela criança ou adolescente e também por parte dos médicos, com o tumor cerebral como causa 
da cefaléia, felizmente os dados estatísticos apontam, para essa etiologia, uma freqüência menor de $1 \%$ nos serviços especializados no atendimento de pacientes com cefaléia. Vale lembrar que o caso individual de um paciente com cefaléia com características atípicas, que mude de padrão, que apresente desencadeantes como o esforço físico, que o exame neurológico mostre alteração, que responda mal ao tratamento de rotina, pode requerer avaliação de um especialista.

\section{Infecções tropicais}

Neurocisticercose: a neurocisticercose é um importante problema de saúde pública. A neurocisticercose humana é causada pela presença do Cysticercus cellulosae, a forma larvária da Taenia solium. A cefaléia é um sintoma que está presente na maioria das síndromes neurocisticercóticas, como a hipertensão intracraniana, a meningite e a meningoencefalite. As características dessa cefaléia, em geral, confundem-se com a enxaqueca ou com a cefaléia tipo tensão. Esses pacientes costumam apresentar quadros de cefaléia intratáveis com sintomáticos ${ }^{2}$. $\mathrm{O}$ tratamento específico deve ser feito pelo especialista.

Malária: síndrome meningítica que pode ser observada no curso de uma crise de malária. Entre os plasmódios que infectam o homem, o $P$. falciparum é o que pode produzir manifestações cerebrais. A cefaléia pode aparecer devido a alterações vasculares, hipóxia, microinfartos, hemorragias, inflamação, e a hipertensão intracraniana, pelo edema cerebral ${ }^{2}$. O tratamento e o acompanhamento devem ser feitos pelo especialista.

\section{CEFALÉIA ASSOCIADA A SUBSTÂNCIAS OU À SUA RETIRADA}

São necessários estudos duplo-cegos controlados com placebo para estabelecer que uma substância realmente induz à cefaléia. Isso foi claramente demonstrado em dois estudos nos quais pacientes relataram cefaléia após ingestão de chocolate amargo ou de aspartame. Em ambos os casos, a cefaléia foi igualmente freqüente após placebo. Teve comprovação a cefaléia induzida pela ingestão de nitratos, nitritos, glutamato monossódico, álcool, ergotamina, analgésicos e pela inalação de monóxido de carbono. Para o diagnóstico de que a retirada de uma substância seja responsável por cefaléia, é necessário que ela apareça após o uso de uma dose mínima e que ocorra quando essa substância é total ou quase totalmente eliminada. Isso ocorre com a supressão do álcool, da ergotamina, da cafeína e de narcóticos.

\section{CEFALÉIA ASSOCIADA À INFE CÇÃO NÃO-CEFÁLICA}

Refere-se às infecções virais, às infecções bacterianas e a outras infecções.

\section{CEFALÉIA ASSOCIADA A DISTÚRBIO METABÓLICO}

Critérios diagnósticos:

- Sinais e/ou sintomas de distúrbio metabólico.

- Confirmação laboratorial quando especificado para uma determinada subforma.

- Intensidade da cefaléia e/ou da sua freqüência está relacionada com as variações do distúrbio metabólico em um determinado período de tempo específico.

- A cefaléia desaparece dentro de 7 dias, após a normalização do estado metabólico.

Os quadros são: cefaléia por hipóxia produzida de altitudes elevadas, por doença pulmonar, por hipercapnia, por hipoglicemia e cefaléia da diálise.

\section{CEFALÉIA OU DOR FACIAL ASSOCIADA A DISTÚRBIO DO CRÂNIO, PESCOÇO, OLHOS, ORELHAS, SEIOS PARANASAIS, DENTES OU OUTRAS ESTRUTURAS FACIAIS OU CRANIANAS}

\section{Causas oculares}

As patologias oculares associadas à cefaléia são: o glaucoma agudo, os erros de refração não corrigidos, como hipermetropia, astigmatismo, presbiopia e uso incorreto de óculos; a heteroforia ou heterotropia e a neurite óptica. O importante, nesses quadros, é que os dados da história clínica sugiram uma estreita relação da dor de cabeça com o uso da visão e a melhora quando esse desencadeante é contornado.

\section{Causas temporomandibulares}

A dor da articulação temporomandibular ou dos tecidos a ela relacionados é comum, mas raramente é devida a um distúrbio orgânico definido. Além disso, quando distúrbios orgânicos são encontrados, tais como os da artrite reumatóide, a dor significativa ou a disfunção 
importante não costumam ocorrer. Sem dúvida, a causa mais freqüente de dor proveniente dessa articulação é miofacial, devido à disfunção oromandibular e à tensão.

\section{Causas nasofaríngeas}

A sinusite aguda pode acompanhar-se de cefaléia.

Os critérios para esse diagnóstico são:

- descarga nasal purulenta espontânea ou evidenciada por sucção;

- achados patológicos em um ou mais dos seguintes exames: raios $\mathrm{X}$, tomografia computadorizada, ressonância magnética e transiluminação;

- início simultâneo da cefaléia e da sinusite;

- localização da cefaléia: 1 - na sinusite frontal aguda, a cefaléia é localizada diretamente sobre o seio e pode irradiar-se para o vértice ou para atrás dos olhos; 2 na sinusite maxilar aguda, a cefaléia é localizada diretamente sobre a área antral e pode irradiar-se para os dentes superiores ou para a fronte; 3 - na etmoidite aguda, a cefaléia é localizada entre e atrás dos olhos e pode se irradiar para a área temporal; 4 - na esfenoidite aguda, a cefaléia é localizada na região occipital, no vértice, na região frontal ou atrás dos olhos.

- a cefaléia desaparece após o tratamento da sinusite aguda.

Tem sido freqüente a suposição do diagnóstico de cefaléia por sinusopatia, com as consequientes medidas de investigação, bem como tratamento ou encaminhamento ao especialista. Nos serviços de atendimento especializado em otorrinolaringologia, a cefaléia associada à sinusopatia aparece em menos de $30 \%$ dos casos. O tratamento é feito com quimioterápicos cabíveis para o tipo de infecção.

\section{SUMMARY}

\section{Secondary Headache in Childhood}

An overview of secondary or symptomatic headaches in children are presented following the International Headache Society Classification. The author's conceptual definition of secondary or symptomatic headaches is presented and peculiarities of the main clinical aspects in infancy are considered for diagnosis regarding clinic experience. Are, also made brief comments on how to conduct the cases.

\section{KEYWORDS}

Headache in children, secondary headache on childhood, symptomatic headache on childhood.

\section{Referências}

1. IHS - International Headache Society. Headache Classification Commitee. Classification and diagnostic criteria for headache disorders, cranial neuralgias and facial pain. Cephalalgia, 8(suppl. 7):1-96, 1988.

2. Carvalho, DS. Symptomatic headaches: tropical infections. In: Headache and migraine in childhood and adolescence. Guidetti V, Russel G, Sillanpää M, Winner P (eds.). Martin Dunitz Publishers, London, (aceito para publicação em 2000).

3. Barlow CF. Headaches and migraine in childhood. Clinics in developmental medicine no 91. Philadelphia, Spastics International Medical, 1984. 288p.

4. Hockaday JM, Barlow CF. Headache in children. In: Olesen J, Tfelt-Hansen P, Welch KMA (eds.). The Headaches. New York, Raven Press, 1993, pp. 795-808.

5. Hockaday JM. Headaches in children. In: Vinken PJ, Bruyn GW, Klawans HL (eds.). Headache. Amsterdam, Elsevier, 1986.

6. Lewis DW, Middlebrook MT, Mehallick L, Rauch TM, Deline C Thomas EF. Pediatric headaches: what do the children want? Headache, 36:224-30, 1996.

7. Massiou $\mathrm{H}$. What is lacking in the treatment of paediatric and adolescent migraine? Cephalalgia, 17(suppl. 17):21-4, 1997.

8. Antonaci $F$. What is the role of international societies and what could they do better? Cephalalgia, 17(suppl. 17):25-8, 1997.

9. Winner P. Headache in children: diagnostic problems and emerging treatments. Cephalalgia, 17:228, 1997.

10. Sillanpää M, Piekkala $P$, Kero P. Prevalence of headache at preschool age in an unselected population. Cephalalgia, $11: 239-42,1991$.

11. Waters WE. Community studies of the prevalence of headache. Headache, 9:178-86, 1974.

12. Olesen J. The secondary headaches: introduction. In: The headaches. Olesen J, Tfelt-Hansen P, Welch KMA (eds.). Philadelphia, Lippincott Williams \& Wilkins, 2000, pp. 763-4.

13. Forsyth PA, Posner JB. The secondary headaches: intracranial neoplasms. In: The headaches. Olesen J, TfeltHansen P, Welch KMA (eds.). Philadelphia, Lippincott Williams \& Wilkins, 2000, pp. 849-59.

\section{Endereço para correspondência:}

Rua Pedro de Toledo, 980 - cj. 33

Vila Clementino - São Paulo, SP

Fone/Fax: (0XX11) 574-6843

E-mail: deusveni@provida.org.br 